\title{
Phononic and magnetic excitations in the quasi-one-dimensional Heisenberg antiferromagnet $\mathrm{KCuF}_{3}$
}

\author{
V. Gnezdilov ${ }^{1,2}$, J. Deisenhofer ${ }^{3}$, P. Lemmens ${ }^{2}$, D. Wulferding ${ }^{2}$, O. Afanasiev ${ }^{1}$, P. Ghigna ${ }^{4}$, \\ A. Loidl ${ }^{4}$, and A. Yeremenko ${ }^{1}$ \\ ${ }^{1}$ B. Verkin Institute for Low Temperature Physics and Engineering of the National Academy of Sciences of Ukraine \\ 47 Lenin Ave., Kharkov 61103, Ukraine \\ E-mail: gnezdilov@ilt.kharkov.ua \\ ${ }^{2}$ Institute for Condensed Matter Physics, Braunschweig University of Technology, \\ D-38106 Braunschweig, Germany \\ ${ }^{3}$ Experimental Physics V, Center for Electronic Correlations and Magnetism, University of Augsburg \\ D-86135 Augsburg, Germany \\ ${ }^{4}$ Dipartimento di Chimica Fisica, Universita di Pavia, 1-27100 Pavia, Italy
}

Received December 15, 2011

\begin{abstract}
The Raman-active phonons and magnetic excitations in the orbitally ordered, quasi-one-dimensional Heisenberg antiferromagnet $\mathrm{KCuF}_{3}$ are studied as a function of temperature in the range between 3 and $290 \mathrm{~K}$ in various scattering configurations. The low-energy $E_{g}$ and $B_{1 g}$ phonon modes show an anomalous softening $(\sim 25 \%$ and $\sim 13 \%$ ) between room temperature and the characteristic temperature $T_{S}=50 \mathrm{~K}$. In this temperature range a freezing-in of $\mathrm{F}^{-}$ion dynamic displacements is proposed to occur. In addition, the $E_{g}$ mode at about $260 \mathrm{~cm}^{-1}$ clearly splits below $T_{S}$. The widths of the phonon lines above $T_{S}$ follow an activated behavior with an activation energy of about $50 \mathrm{~K}$. Our observations clearly evidence a reduction of the lattice symmetry below $T_{S}$ and indicate a strong coupling of lattice, spin, and orbital fluctuations for $T>T_{S}$. A strongly polarization dependent quasielastic scattering is observed at temperatures above $T_{N}=39 \mathrm{~K}$ due to magnetic-energy fluctuations. For temperatures below $T_{N}$ a rich spectrum of additional modes is observed, with different lineshape, polarization and temperatures dependence. We attribute them to longitudinal and transverse magnetic modes as well as to a continuum of magnetic excitations.
\end{abstract}

PACS: 63.20.-e Phonons in crystal lattices;

75.25.-j Spin arrangements in magnetically ordered materials (including neutron and spin-polarized electron studies, synchrotron-source x-ray scattering, etc.);

75.25.Dk Orbital, charge, and other orders, including coupling of these orders;

75.50.-y Studies of specific magnetic materials;

78.30.-j Infrared and Raman spectra.

Keywords: Raman scattering, low-dimensional magnetic systems, dynamical Dzyaloshinsky-Moriya interaction, magnetic excitations.

\section{Introduction}

$\mathrm{KCuF}_{3}$ has long been known as a paradigm for an orbitally ordered system where a cooperative Jahn-Teller (JT) distortion is strongly competing with the electronic degrees of freedom as the driving force behind the orbital order [1-4]. Recently, realistic band structure calculations highlighted $\mathrm{KCuF}_{3}$ as a benchmark system for modeling structural relaxation effects due to electronic correlations
$[5,6]$ and for revealing the influence of electronic superexchange on the orbital ordering (OO) [7]. The compound seems to be orbitally ordered throughout its solid phase, but shows long-range A-type antiferromagnetic (AFM) ordering only below $T_{N}=39 \mathrm{~K}$. In literature an orbital ordering temperature of about $800 \mathrm{~K}$ is often evoked in this system, but astonishingly experimental evidence for a transition at this temperature seems to be evasive. Early on it was reported that between 670 and $720 \mathrm{~K}$ an irreversible 
transition takes place [8]. The paramagnetic (PM) susceptibility is described by a Bonner-Fisher law with an exchange constant $J=190 \mathrm{~K}$ [1], indicating that the compound is a good realization of a one-dimensional (1D) spin chain in the PM regime. Inelastic neutron scattering (NS) studies reveal a spinon-excitation continuum, a clearly 1D quantum phenomenon, existing also below the Néel temperature $[9,10]$. From a structural point of view the relatively high tetragonal symmetry [11-16] $\left(D_{4 h}^{18}-I 4 / \mathrm{mcm}\right)$ makes $\mathrm{KCuF}_{3}$ one of the simplest systems to study. There exist a second structural polytype $\left(D_{4 h}^{5}\right)$ with a different arrangement of the $\mathrm{F}$ distortions within the $c$ plane [14]. This phase that may exist to a few $\%$ in every sample has different physical properties in the sense of magnetic anisotropies and ordering temperature $\left(T_{N}=22 \mathrm{~K}\right)$. Nevertheless, the established symmetry assignment of the $D_{4 h}^{18}$ phase has been questioned by an x-ray diffraction investigation [17] which suggests the existence of orthorhombic distortions in $\mathrm{KCuF}_{3}$ at room temperature with $D_{2}^{4}$ symmetry. A low-temperature Raman scattering (RS) study [18] reveals a difference of spectra measured in $x z$ and $y z$ polarization and anomalously broad linewidths of the stretching modes, which are interpreted as evidence of symmetry lower than $D_{4 h}^{18}$ also below the Néel temperature. Although orthorhombic distortions are involved for explaining the electron spin resonance (ESR) properties of $\mathrm{KCuF}_{3}$ [19], discrepancies remain for the analysis of recent NQR [20], AFM resonance [21], and further experimental and theoretical findings $[22,23]$. Besides, in $\mathrm{x}$-ray resonant scattering $[24,25]$ of the $\mathrm{OO}$ in $\mathrm{KCuF}_{3}$ indications for a coupling of lattice and magnetic degrees of freedom above $T_{N}$ are found. Only recently, the ESR properties for $T>T_{N}$ could be successfully explained within the tetragonal symmetry by assuming a dynamical Dzyaloshinsky-Moriya (DM) interaction related to strong oscillations of the bridging $\mathrm{F}^{-}$ions perpendicular to the crystallographic $c$ axis [26]. It is argued that these dynamic distortions freeze in at a temperature $T_{S}=50 \mathrm{~K}$, leading to an effectively lower symmetry and the occurrence of exciton-magnon sidebands in optical absorption experiments [27].

Here we report on a detailed study of:

(i) the temperature dependence of the Raman-active phonons in a $\mathrm{KCuF}_{3}$ single crystal, tracking the symmetry reduction during the anticipated freezing of the dynamic distortion at $T_{S}=50 \mathrm{~K}$ and the Néel ordering at $T_{N}=39 \mathrm{~K}$;

(ii) a complete data set of the magnetic excitations in this compound collected for the first time by using Raman scattering.

We find a large softening of the lowest lying $E_{g}$ mode and the $B_{1 g}$ mode by $25 \%$ and $13 \%$ between room temperature and $T_{S}$, respectively. The linewidth and the integrated intensity of these modes also exhibit anomalies at $T_{S}$ and $T_{N}$. Moreover, the $E_{g}$ mode at about $260 \mathrm{~cm}^{-1}$ clearly splits below $T_{S}$ evidencing the existence of an antiferrodistortive lattice instability in $\mathrm{KCuF}_{3}$ which leads to a symmetry reduction at $T_{S}=50 \mathrm{~K}$ prior to magnetic ordering.

At low temperatures (below $T_{N}$ ) magnetic excitations characteristic of 3D and 1D magnetic correlations are observed simultaneously in our Raman experiment. The energy ranges of these phases are separated by another region where the crossover from $1 \mathrm{D}$ to $3 \mathrm{D}$ behavior occurs.

\section{Experimental details}

The single crystal was oriented by Laue diffraction, cut along the (110) pseudocubic plane and mechanically polished to optical quality. Details on crystal growth are described in Ref. 25. The sample has previously been investigated by ESR and optical spectroscopy [26,27]. The Raman spectra were obtained with two different experimental setups and in two geometries of experiment: (i) a DILOR XY triple spectrometer with a liquid-nitrogencooled CCD detector (quasi-backscattering geometry) and (ii) a U1000 high-resolution double spectrometer with RCA 31034A photomultiplier (right-angle scattering geometry). The $647 \mathrm{~nm} \mathrm{Ar} / \mathrm{Kr}$ ion (5 mW output power), the $532.1 \mathrm{~nm}$ solid-state ( $5 \mathrm{~mW}$ output power), and the $632.8 \mathrm{~nm} \mathrm{He}-\mathrm{Ne}$ ( $25 \mathrm{~mW}$ output power) lasers were used for excitation in these two setups. Temperature dependencies were obtained in optical gas-flow cryostats and in a closed cycle cryostat by varying temperature from $3 \mathrm{~K}$ to a room temperature.

\section{Experimental results and discussion}

\subsection{Raman phonons}

The polarized phonon Raman spectra of single crystalline $\mathrm{KCuF}_{3}$ were taken in $y y, z z, x y, x z$, and $y z$ scattering configurations. The number of lines and the selection rules are fully consistent with the theoretically expected Raman-active normal modes [18] of $\mathrm{KCuF}_{3}$ with tetragonal $D_{4 h}^{18}$

$$
\Gamma_{\mathrm{Ram}}=A_{1 g}(y y, z z)+B_{1 g}(y y)+2 B_{2 g}(x y)+3 E_{g}(x z, y z) .
$$

The three lines observed in Raman spectra at $290 \mathrm{~K}$ in both the $x z$ and $y z$ spectra correspond to the three $E_{g}$ modes. The line observed with different intensities in $y y$ and $z z$ spectra is identified as the $A_{1 g}$ mode. The intense line observed only in the $y y$ spectrum can be assigned to the $B_{1 g}$ mode. Finally, the two lines in the $x y$ spectra are the two $B_{2 g}$ modes. At room temperature all lines have a Lorentzian lineshape. The observed spectra and mode assignments are in agreement with previously reported data at $10 \mathrm{~K}$ [18]. A direct comparison of our data at $4 \mathrm{~K}$ and $290 \mathrm{~K}$ with Ref. 18 and theoretical estimates [28] is presented in Table 1. In general, there is a good agreement between the corresponding values except for the $B_{2 g}(1)$ mode with a frequency of $240.4 \mathrm{~cm}^{-1}$ observed in our experiments in contrast to a somewhat higher frequency of $265.8 \mathrm{~cm}^{-1}$ in Ref. 18. The second discrepancy is that the lines assigned to $E_{g}(1,2)$ and 
$B_{1 g}$ are almost two times broader in the low-temperature Raman spectra of Ref. 18. We can relate these discrepancies to different quality of the studied samples.

Table 1. Frequencies and linewidths of the observed Raman

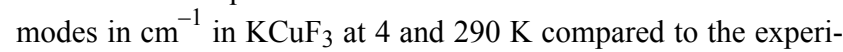
mental values reported in Ref. 18 at $10 \mathrm{~K}$ and calculations from Ref. 28

\begin{tabular}{|c|c|c|c|c|c|c|c|}
\hline \multirow[t]{2}{*}{ Mode } & \multicolumn{4}{|c|}{ Frequency, $\mathrm{cm}^{-1}$} & \multicolumn{3}{|c|}{ Linewidth, $\mathrm{cm}^{-1}$} \\
\hline & $\begin{array}{l}\frac{v}{8} \\
\text { নे }\end{array}$ & $\stackrel{\forall}{\sigma}$ & $\begin{array}{l}\infty \\
\underset{\Xi}{\Xi} \\
0\end{array}$ & 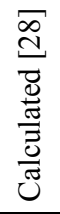 & $\begin{array}{l}\stackrel{v}{8} \\
\stackrel{\text { iे }}{2}\end{array}$ & $\frac{\forall}{\gamma}$ & $\begin{array}{l}\underset{\infty}{\Xi} \\
\stackrel{\infty}{\Xi} \\
0\end{array}$ \\
\hline$A_{1 g}$ & 367.3 & 373.5 & 374.8 & 398 & 23.1 & 4.9 & 9.2 \\
\hline$B_{1 g}$ & 81.6 & 70.9 & 72.8 & 100 & 7.1 & 0.9 & 1.6 \\
\hline$B_{2 g}(1)$ & 240.4 & 245.2 & 265.8 & 259 & 30.8 & 8.7 & 7.0 \\
\hline$B_{2 g}(2)$ & 554.8 & 561.3 & 563.0 & 586 & 22.6 & 9.1 & 9.1 \\
\hline$E_{g}(1)$ & 63.0 & 47.4 & 53.2 & 50 & 5.8 & 0.7 & 3.0 \\
\hline$E_{g}(2)$ & 262.9 & 260.8 & 261.6 & 136 & 7.5 & 1.7 & 3.0 \\
\hline$E_{g}(3)$ & 132.3 & 129.3 & 131.2 & 268 & 7.5 & 1.6 & 1.6 \\
\hline
\end{tabular}

In the following we will focus on the temperature dependence of the phonon modes in $\mathrm{KCuF}_{3}$ and mostly $E_{g}(1), E_{g}(2)$ and $B_{1 g}$. In Figs. 1-3 we show the temperature dependent parameters (frequency, linewidth, and integrated intensity, respectively) for the five phonon modes. The phonon lines of $A_{1 g}$ and $B_{2 g}$ symmetry have large

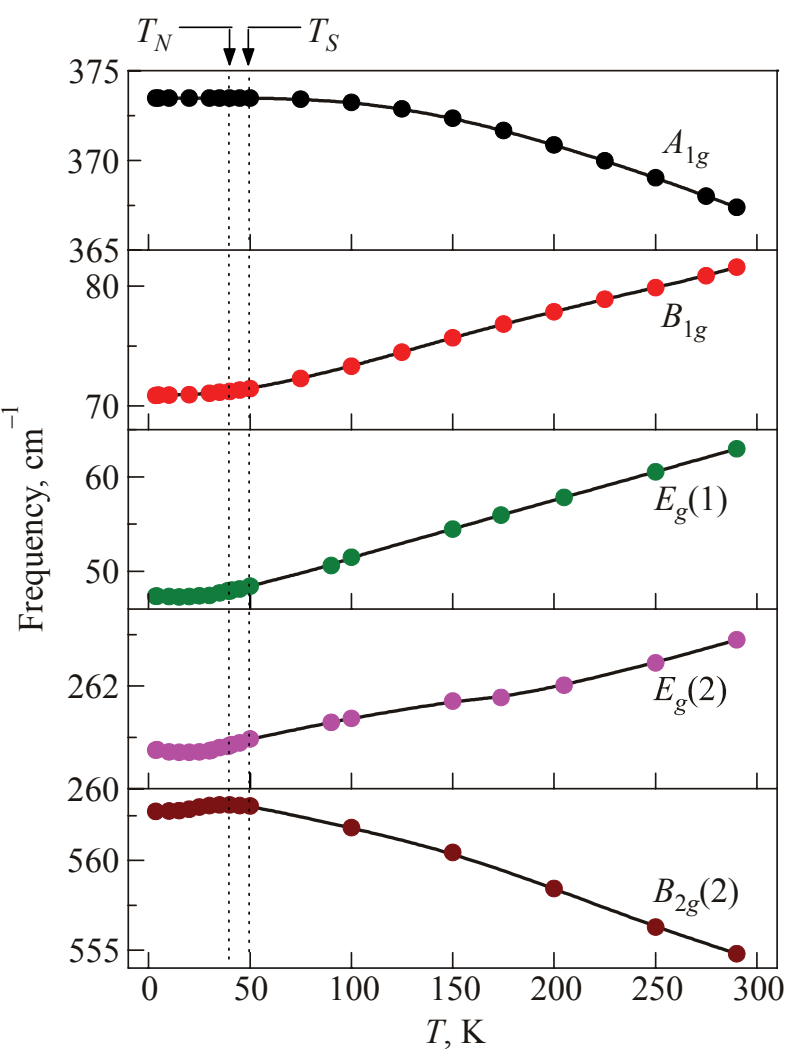

Fig. 1. Temperature dependence of the eigenfrequency of the phonon modes in $\mathrm{KCuF}_{3}$. Solid lines are guides to the eye. linewidths in comparison with the other modes. These modes, aside from their broadened lineshape, show no frequency vs. temperature anomalous behavior. In the full temperature range they exhibit a hardening of $1-2 \%$. The temperature dependence of the $A_{1 g}$ phonon mode eigenfrequency (see Fig. 1) fits very well to the equation

$$
\omega_{i}(T)=\omega_{0 i}\left[1-\frac{c_{i}}{\exp \left(h c \omega_{\mathrm{av}} / k_{B} T\right)-1}\right],
$$

including anharmonic effects [29]. Here $\omega_{0 i}$ indicates the eigenfrequency of the phonon in the absence of spinphonon coupling at $0 \mathrm{~K}, c_{i}$ is a mode dependent scaling factor, and $\omega_{\mathrm{av}}$ is the arithmetic average of the IR- and Raman phonon frequencies at room temperature.

The $A_{1 g}$ phonon mode linewidth is shown in Fig. 2 together with the expected $T$ dependence according to a cubic anharmonicity in second order, i.e., [30,31]

$$
\Gamma(T)=\Gamma_{0}\left[1+\frac{2}{\exp \left(h c \omega_{0} / 2 k_{B} T\right)-1}\right],
$$

with $\Gamma_{0}=4.88 \mathrm{~cm}^{-1}$ and $\omega_{0}=373.5 \mathrm{~cm}^{-1}$. A large $A_{1 g}$ phonon peak broadening is observed for temperatures above $\sim 50 \mathrm{~K}$. This anomalous broadening of the hard $A_{1 g}$

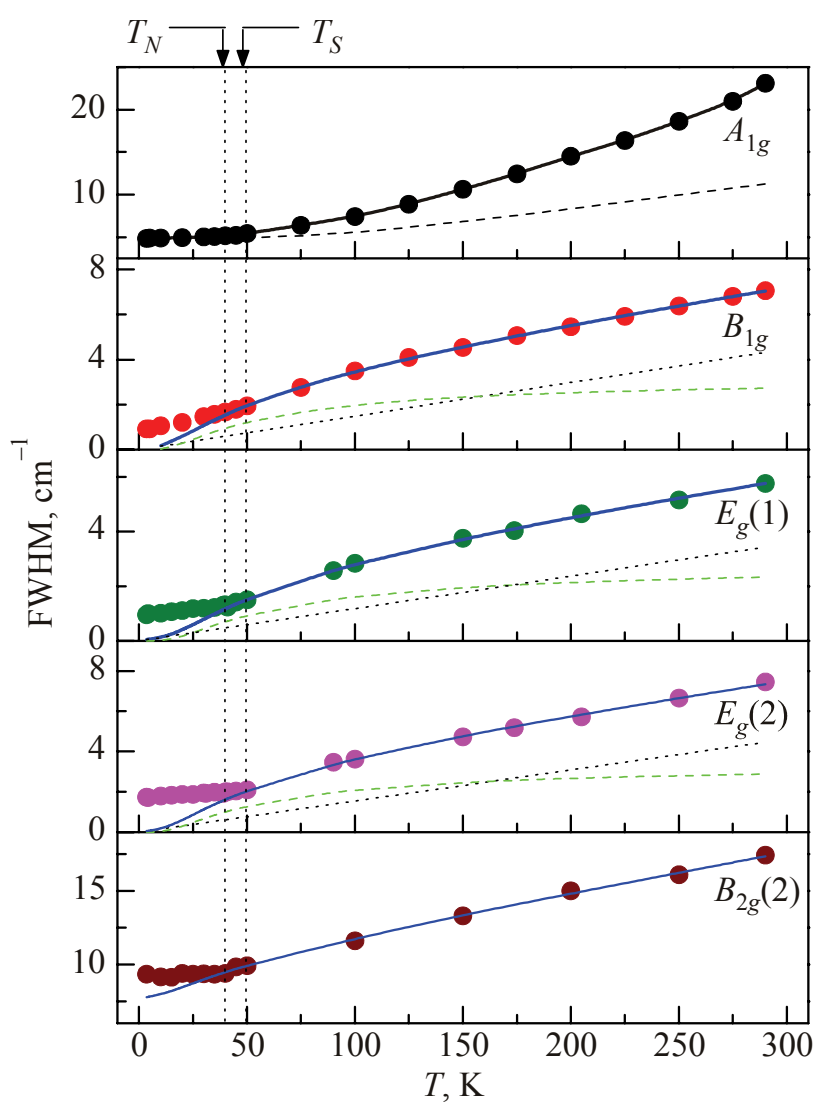

Fig. 2. Temperature dependence of the linewidth (full width at half maximum) of the phonon modes in $\mathrm{KCuF}_{3}$. The dashed line in the " $A_{1 g}$ " panel is a fit using Eq. (3). In the " $B_{1 g}$ ", " $E_{g}(1)$ ", and " $E_{g}(2)$ " panels FWHMs are given with a fit using Eq. (9); dashed and dotted lines: first and second terms in Eq. (9), respectively. 
phonon mode can be understood assuming structural fluctuations at $T>50 \mathrm{~K}$. Such structural fluctuations decrease the phonon mean lifetime and account for the anomalous phonon broadening observed in $\mathrm{KCuF}_{3}$.

The $E_{g}(1)$ mode reportedly exhibits a weak splitting at $10 \mathrm{~K}$ only when measured in $y z$ configuration. In contrast, the $E_{g}(2)$ mode shows a splitting only when measuring in $x z$ configuration. The $E_{g}(3)$ mode, which corresponds to a vibration of $\mathrm{K}^{+}$ions, shows no splitting in either of the two configurations [18]. The $E_{g}(1)$ and $E_{g}(2)$ modes correspond to shearing vibrations of the $\mathrm{F}^{-}$ions which involve a displacement of the fluorine ions away from the $\mathrm{Cu}-\mathrm{F}-\mathrm{Cu}$ bonding lines, while the $B_{1 g}$ mode corresponds to a tilting motion of the $\mathrm{F}^{-}$ions around the central $\mathrm{Cu}$ atom. As such displacements are thought to be the origin of the dynamical DM interaction which allows to understand the ESR and antiferromagnetic resonance properties, we expect that these modes are strongly related to the proposed freezing of the dynamic fluorine displacements below $T_{S}=50 \mathrm{~K}$ [26,27].

Indeed, when looking at the Raman data of the $E_{g}(1)$ and $E_{g}(2)$ vibrational modes in $y z$ configuration shown in Fig. 4, an anomalous softening of both modes is observed for $T>50 \mathrm{~K}$. While the frequency shift of the $E_{g}(2)$ is only about $2 \mathrm{~cm}^{-1}$, the low-energy $E_{g}(1)$ mode exhibits a frequency shift of about $16 \mathrm{~cm}^{-1}$. This corresponds to a softening of 1 and $25 \%$ with respect to the room temperature eigenfrequency.

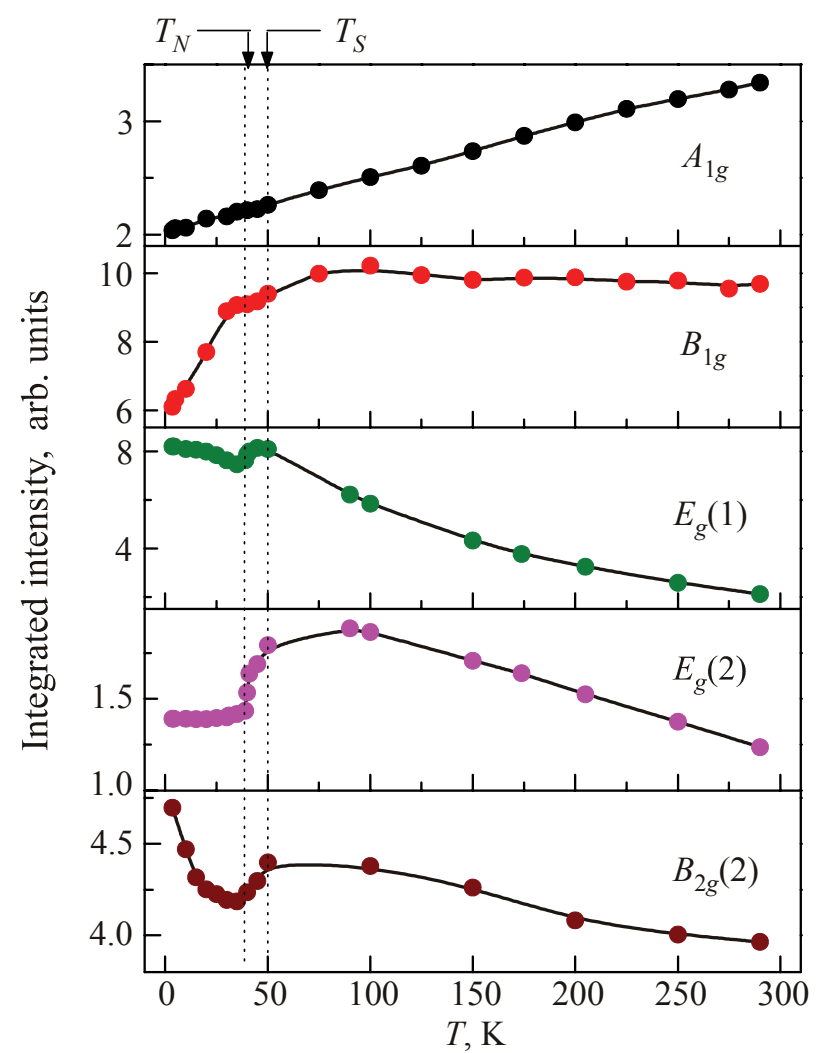

Fig. 3. The Bose corrected integrated intensity of the phonon modes in $\mathrm{KCuF}_{3}$ (solid lines are guides to the eye).
To discuss the origin of the observed effect, we first assume that this mode softening is the precursor of a structural phase transition [32] with a $\omega_{E_{g}}^{2} \propto\left(T-T_{c}\right)$ soft mode behavior, where $T_{c}$ is the phase transition temperature. Indeed the frequency data for the $E_{g}(1)$ mode in $\mathrm{KCuF}_{3}$ can be fitted excellently for $T>50 \mathrm{~K}$ using

$$
\omega_{E_{g}}^{2}=\alpha T+\omega_{0}^{2},
$$

with $\alpha=8.93 \mathrm{~cm}^{-2} / \mathrm{K}$ and $\omega_{0}=45.53 \mathrm{~cm}^{-1}$. Extrapolating $\omega_{E_{g}(1)}^{2}$ to zero one can obtain a virtual transition temperature $T_{v}=-334 \mathrm{~K}$. Although the negative sign indicates that the occurrence of the structural phase transition is very unlikely, the energy scale of this virtual transition temperature is close to the orbital-ordering transition temperature $T_{o o} \sim 350 \mathrm{~K}$ calculated by assuming a purely electronic superexchange mechanism [7].

Similar to the $E_{g}(1)$ mode, both $E_{g}(2)$ and $B_{1 g}$ modes exhibit a softening at $T>50 \mathrm{~K}$ (see Figs. 1 and 4) with $\alpha=$ $=3.99 \mathrm{~cm}^{-2} / \mathrm{K}$ and $T_{v}=-16858 \mathrm{~K}$ for the $E_{g}(2)$ and $\alpha=$ $=6.43 \mathrm{~cm}^{-2} / \mathrm{K}$ and $T_{v}=-743 \mathrm{~K}$ for the $B_{1 g}$ mode. While for the $B_{1 g}$ with a softening of about $13 \%$ with respect to room temperature the virtual transition temperature is still reasonable, the value for the $E_{g}(2)$ mode appears not to be physically meaningful due to the moderate softening of only $1-2 \%$. Note that in other fluorides with rutile structure like $\mathrm{MnF}_{2}, \mathrm{NiF}_{2}$, and $\mathrm{FeF}_{2}$ virtual transition temperatures of $-1240 \mathrm{~K},-1700 \mathrm{~K}$, and $-1780 \mathrm{~K}$ have been derived from the softening of Raman modes, respectively [33-35].

In magnetic materials, the frequency change of a phonon $i$ with temperature can be written as [36-40]

$$
\Delta \omega_{i}(T)=\left(\Delta \omega_{i}\right)_{\text {latt }}+\left(\Delta \omega_{i}\right)_{\text {ren }}+\left(\Delta \omega_{i}\right)_{s-\mathrm{ph}}+\left(\Delta \omega_{i}\right)_{\mathrm{anh}}
$$

The first term, $\left(\Delta \omega_{i}\right)_{\text {latt }}$, is the contribution to the phonon frequency due to the lattice expansion/contraction. For a nearly cubic lattice it can be roughly estimated by the Grüneisen law:

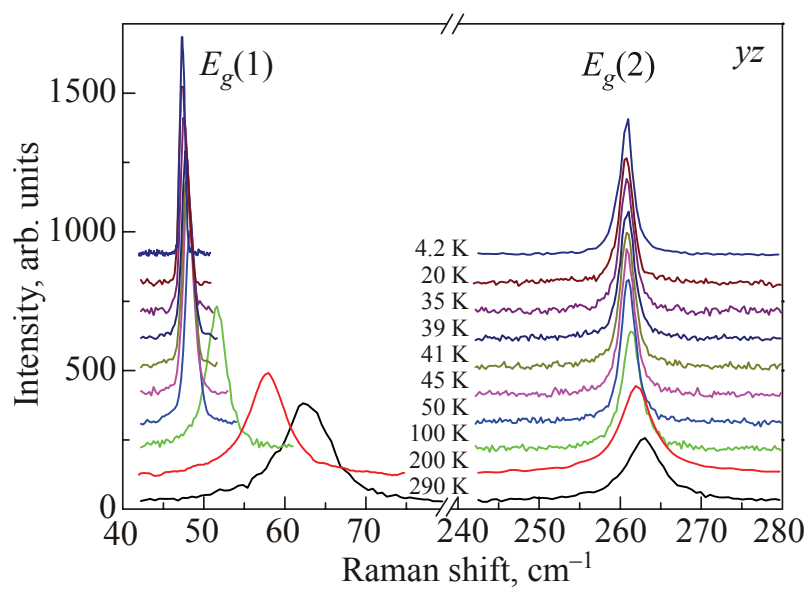

Fig. 4. Temperature dependence of the $E_{g}(1)$ and $E_{g}(2)$ modes obtained in $y z$ scattering geometry. 


$$
\left(\frac{\Delta \omega_{i}}{\omega_{i}}\right)_{\text {latt }}=-\gamma_{i}\left(\frac{\Delta \mathrm{V}}{\mathrm{V}}\right)
$$

where $\gamma_{i}$ is the Grüneisen parameter for the phonon mode $i$. Equation (6) is applicable for cubic crystals or for isotropically expanded/contracted lattices. The second term, $\left(\Delta \omega_{i}\right)_{\text {ren }}$, accounts for the effects in the phonon $i$ of a renormalization of the electronic states that may occur near the spin ordering temperature. The third term, $\left(\Delta \omega_{i}\right)_{s-\mathrm{ph}}$, is the spin-phonon contribution, caused by the modulation of the exchange integral by lattice vibrations. Finally, the last term $\left(\Delta \omega_{i}\right)_{\mathrm{anh}}$ is the intrinsic anharmonic contribution, i.e., the anharmonic frequency shift at constant volume.

To analyze the origin of the observed phonon modes behavior in $\mathrm{KCuF}_{3}$ at temperatures above $50 \mathrm{~K}$, we first consider the lattice contribution $\left(\Delta \omega_{i}\right)_{\text {latt }}$. The lattice parameters in $\mathrm{KCuF}_{3}$ show a smooth thermal contraction below $T_{o o}$ (c/a decreases not more than $0.1 \%$ between $T_{N}$ and room temperature [8]) without anomalies at $T_{N}$. Phonons are expected (with regard to $\left.\left(\Delta \omega_{i}\right)_{\text {latt }}\right)$ to shift to higher frequencies with decreasing temperature, as the spring constants of all vibrations tighten at low temperatures. Besides, $\mathrm{KCuF}_{3}$ is an insulator at all temperatures of our experiment. Therefore the carrier-induced vibrational softening (with regard to $\left.\left(\Delta \omega_{i}\right)_{\text {ren }}\right)$ is not a suitable model for this system.

The spin influence on the lattice dynamics has been studied by several authors. In particular, considering a nearest neighbor Heisenberg spin system, Baltensberger and Helman related the frequency shift of a given phonon mode to the magnetic energy of the system [36-40],

$$
\Delta \omega_{s-\mathrm{ph}} \approx \lambda\left\langle s_{i} \cdot s_{j}\right\rangle,
$$

where $\lambda$ is the spin-phonon coupling constant and $\left\langle s_{i} \cdot s_{j}\right\rangle$ represents the spin-spin correlation function. In the paramagnetic state the coupling parameter, $\lambda$ can be determined if the frequency shift of the phonon mode, $\Delta \omega$ and the spin-spin correlation function, $\left\langle s_{i} \cdot s_{j}\right\rangle$ are known. The latter quantity can be directly derived from the measured susceptibility data:

$$
\left\langle s_{i} \cdot s_{j}\right\rangle_{\chi}=\frac{k_{B} T \chi(T)}{N_{A} g^{2} \mu_{B}^{2}}-\frac{s(s+1)}{3} .
$$

Assuming that the spin-phonon coupling shows up in the temperature dependence of the phonon frequencies of $\mathrm{KCuF}_{3}$ in the paramagnetic state, we have estimated $\lambda$ for the $E_{g}(1), E_{g}(2)$, and $B_{1 g}$ modes using our Raman and magnetic susceptibility (not shown here) data. The spinspin correlation function of $\mathrm{KCuF}_{3}$ shows a similar temperature evolution as the $E_{g}(1), E_{g}(2)$, and $B_{1 g}$ mode frequencies: $\left\langle s_{i} \cdot s_{j}\right\rangle$ decreases upon lowering the temperature from 290 to $50 \mathrm{~K}$. Yet, the estimated spin-phonon coupling constants must be very large $\left(\lambda \approx 100,13,75 \mathrm{~cm}^{-1}\right.$ for the $E_{g}(1), E_{g}(2)$, and $B_{1 g}$ modes, respectively) to explain the observed frequency shift with $\left(\Delta \omega_{i}\right)_{s-\mathrm{ph}}$ term alone. However, for correctly describing the observed frequency shift in $\mathrm{KCuF}_{3}$ it is necessary take into account a sum of different terms in Eq. (7), namely, due to AFM and FM exchanges in the magnetic structure. The sign of the frequency shift depends on whether the $\mathrm{Cu}-\mathrm{F}$ bond vibrations are influenced by AFM or FM exchange.

Lattice instabilities in our Raman experiments strongly manifests itself in anomalous phonon linewidths behavior with temperature (see Fig. 2). Above $50 \mathrm{~K}$ the linewidth data for $E_{g}(1), E_{g}(2)$, and $B_{1 g}$ modes can be described by

$$
\Gamma_{\text {tot }}(T)=\Gamma_{\text {anh }}(T)+\Gamma_{r}(T)=A T+B \exp \left(-\frac{U_{r}}{k_{B} T}\right)
$$

where $\Gamma_{\text {anh }}$ is the contribution arising from anharmonic phonon interactions in crystalline solids, with zone center modes decaying into pairs of phonons with equal but opposite wave vectors. $\Gamma_{r}$ is the contribution to the total linewidth arising from the dynamic deviation of the $\mathrm{F}^{-}$ ions away from the $\mathrm{Cu}-\mathrm{Cu}$ bonding lines, $U_{r}$ is a potential barrier, and $A$ and $B$ are constants. The data can be described very well over the temperature range $290-50 \mathrm{~K}$ by Eq. (9) yielding an energy $U_{r}=56\left(E_{g}(1)\right), 49\left(E_{g}(2)\right)$, and $47\left(B_{1 g}\right) \mathrm{K}$ very close to the temperature $T_{S}=50 \mathrm{~K}$ where the dynamic displacements are proposed to become static [27]. Below $T_{S}=50 \mathrm{~K}$ the width of the phonon lines decrease nearly linearly with temperature.

The lattice fluctuations, associated with the dynamic displacement of the apical fluorine ions away from the $c$ axis manifest themselves in an anomalously large thermal displacements parameter [12] and the occurrence of a dynamical DM interaction [26]. As a prerequisite for the latter, the characteristic time of the dynamic distortions must be large compared to the time scale of the exchange interaction and the amplitude of these distortions must be high [26].

In Ref. 41 an essential role of the orbital degree of freedom in governing the dynamical coupling between the spin and lattice degrees of freedom in $\mathrm{KCuF}_{3}$ was grounded. We recall here that a giant softening of the breathing mode in the FMI phase of lightly doped $\mathrm{LaMnO}_{3}$ was interpreted earlier in terms of the presence of fluctuating orbitals as well as strong orbital-lattice coupling [42]. In this mechanism, the frequency shift of a phonon mode relies on whether the respective ion displacements involve an orbital-ordering pattern. Displacement of apical ions in a perovskite structure usually means a tilt of a $\mathrm{CuF}_{6}$ octahedron as a whole [43]. In-plane fluctuations of $\mathrm{F}^{-}$ions influence the spin-spin correlations due to strong spin-lattice coupling mediated through the orbitals. It is known that FM spin ordering causes a softening of the lattice stiffness, while AFM causes a hardening [41,44]. Taking into account both AFM and FM fluctuating in-plane spin-spin 
correlations in $\mathrm{KCuF}_{3}$ over a wide temperature range above $T_{N}$, it is possible that the development of the FM correlations with decreasing temperature leads to the observed phonon modes softening.

In the $\mathrm{KCuF}_{3}$ scenario the dynamical displacement of the fluorine ions freezes with decreasing temperature and becomes static at $T_{S}=50 \mathrm{~K}[26,27]$. Below $50 \mathrm{~K}$ we observe a deviation from this softening behavior and the frequency levels off in the magnetically ordered state.

The Bose corrected integrated intensities of the phonon modes in $\mathrm{KCuF}_{3}$ are shown in Fig. 3. The intensities of the $E_{g}(1), E_{g}(2), B_{1 g}$, and $B_{2 g}$ modes show anomalies at $T_{S}$ and $T_{N}$, reflecting distinct changes of the modes' polarizability at these temperatures.

To get more information about the coupling between the lattice and the orbit degrees of freedom, we measured Raman spectra in the high-frequency region, expecting to observe some features coupled to orbital dynamics. Unlike doped manganites with very complicated high-order excitation spectra, only one broad peak was observed in $\mathrm{KCuF}_{3}$ in $z z$ scattering geometry at energies above the first-order phonons (see Fig. 5). We can tentatively assign this peak at $\sim 1100 \mathrm{~cm}^{-1}$ to two-phonon scattering of the $555 \mathrm{~cm}^{-1}$ $B_{2 g}(2)$ mode, taking into account the multiplication rules of the irreducible representations and the fact that similar changes in frequencies over temperature are observed. The ratio of the second to first order $I^{(2)} / I^{(1)}$ is constant within

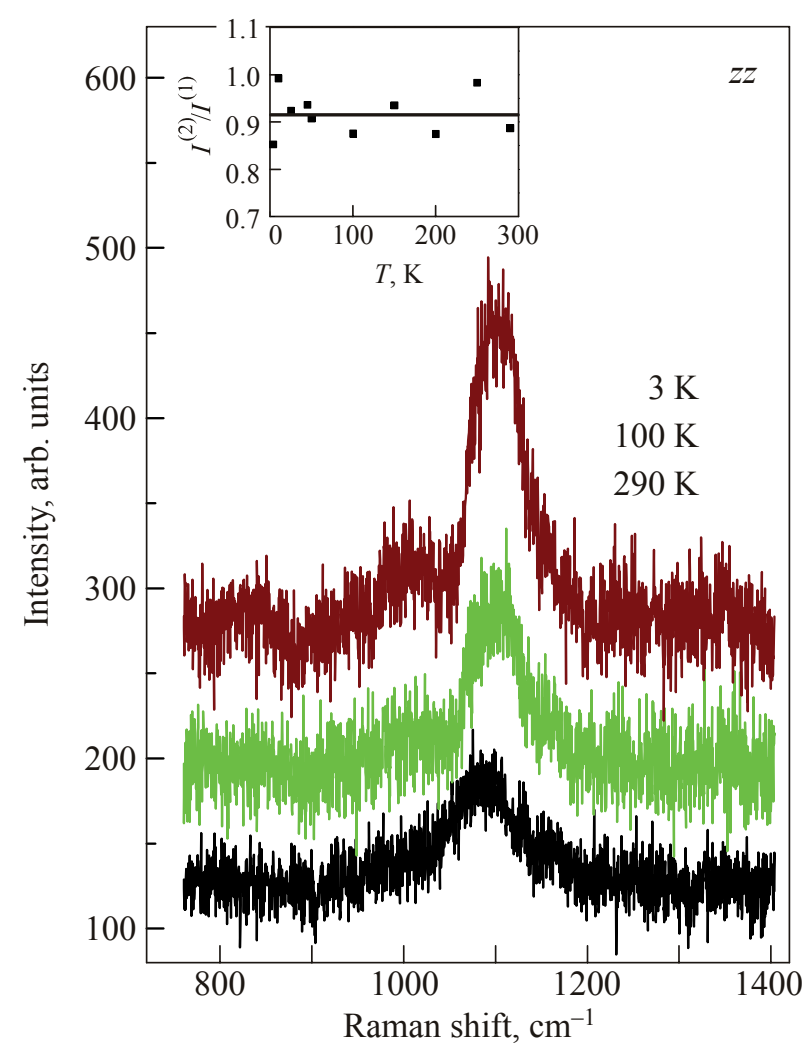

Fig. 5. Second-order Raman spectra of $\mathrm{KCuF}_{3}$. The inset shows the ratio of second- to first-order scattering at $555 \mathrm{~cm}^{-1}$ as a function of temperature. the measured temperature interval. This fact evidences that the second order phonon in $\mathrm{KCuF}_{3}$ couples in some degree to the orbital order and the Franck-Condon mechanism is expected to be active.

Although clear anomalies of phonon modes associated with $T_{S}$ and $T_{N}$ have been observed, we could not observe the splitting of the $E_{g}(1)$ mode in $y z$ configuration reported in Ref. 18 at $10 \mathrm{~K}$. (Only the asymmetry of the $E_{g}(1)$ phonon line develops with the temperature lowering from $T_{S}$ to $T_{N}$.) Hence, we tried to reproduce the reported splitting of the $E_{g}(2)$ mode in $x z$ configuration and trace its temperature dependence. A weak additional mode at the highfrequency side of the $E_{g}(2)$ appears at $T<50 \mathrm{~K}$. As shown in Fig. 6, the appearance of this additional mode coincides with $T_{S}$ suggesting a splitting of the $E_{g}(2)$ mode in agreement with the scenario of a symmetry reduction at $T_{S}$, as suggested previously [27]. Nevertheless, we have to point out that this splitting of about $6 \mathrm{~cm}^{-1}$ is larger than the reported one of about $1 \mathrm{~cm}^{-1}$ and appears on the high-energy flank in contrast to the one reported by Ueda and coworkers [18], which appears on the low-energy side of the original $E_{g}(2)$ mode. These discrepancies can not be easily explained and may be due to the different samples used for our work. We would like to point out that in a recent Raman study a splitting of the $E_{g}(2)$ similar to our data has been reported [41].

A static displacement of fluorine ions away from the $c$ axis at temperatures $T<T_{S}$ assumes the lowering of the $\mathrm{KCuF}_{3}$ crystal symmetry. If the symmetry is lower than $D_{4 h}^{18}$, a removal of the $E_{g}$ modes' degeneracy and the appearance of extra lines in the Raman spectra is expected. The observed splitting of the $E_{g}(2)$ mode in $x z$ configuration confirms this scenario, alone, this information is not sufficient to determine the low-temperature symmetry. Additional evidence has been obtained by x-ray scattering where a splitting of a Bragg reflection associated with $\mathrm{GdFeO}_{3}$ type distortions has been found below $50 \mathrm{~K}$ [41].

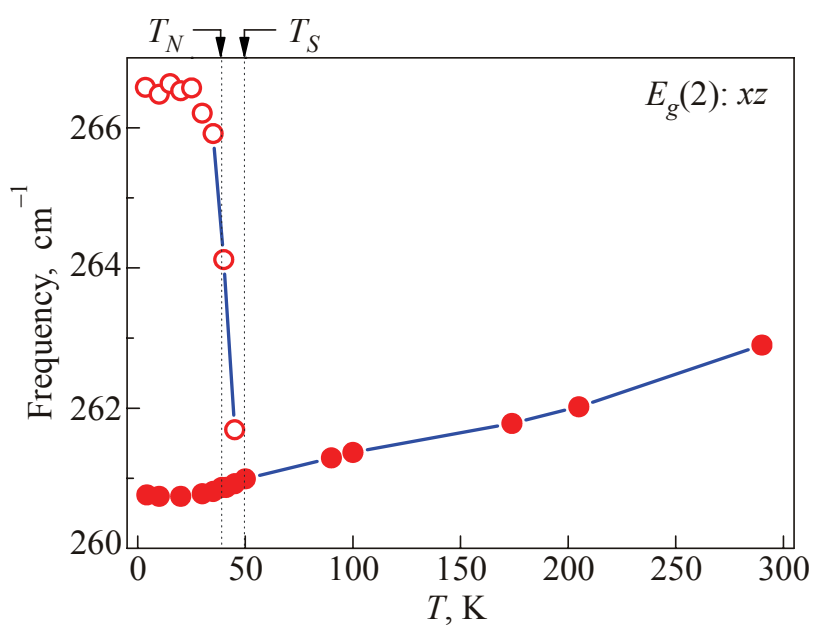

Fig. 6. Temperature dependence of the eigenfrequency of the $E_{g}(2)$ and the split-off mode below $T_{S}$ in $x z$ scattering geometry. Lines are drawn to guide the eye. 
To summarize, we believe that the softening of certain phonon modes in $\mathrm{KCuF}_{3}$ is connected with the simultaneous and coupled change in magnetic-structural-orbital sectors upon cooling. In this respect it is worth highlighting that in $\mathrm{KCuF}_{3}$ the spin-spin relaxation time measured by the ESR linewidth can only be explained by assuming dynamic lattice distortion of the type associated with the anomalous Raman modes [26].

\subsection{Magnetic excitations}

To date, very comprehensive neutron scattering experimental and theoretical work has been directed toward the spin dynamics in $\mathrm{KCuF}_{3}$. The dynamical response for all energy, wave vector, and temperature scales has been sampled and the magnetic crossover diagram (see Fig. 4 in Ref. 9), which shows that the system clearly has aspects of both 1D and 3D spin-1/2 magnetism, has been constructed. It is therefore striking that corresponding investigations using light scattering are still incomplete despite the rich information that light scattering can give [45]. We will omit from our discussion quasielastic scattering due to fluctuations of the spin energy density. This effect has been described previously in $\mathrm{KCuF}_{3}$ by Yamada and Onda [46]. It is a very general feature of low-dimensional spin systems [45] and observed for intrachain polarization, corresponding to $z z$ configuration. Below we report a complete data set of the magnetic excitations in $\mathrm{KCuF}_{3}$ collected for the first time via Raman scattering.

Upon cooling, a broad quasielastic background of scattering is replaced by a more structured spectrum in a wide energy region. At temperatures close and below the 3D ordering temperature the phonon lines in $x x, y y$, and $z z$ Raman spectra of $\mathrm{KCuF}_{3}$ are superimposed by a wider, structured background (Fig. 7). The large width of the observed signal distinguishes it from the sharp phonon lines. Referring to results of numerous neutron scattering inves-

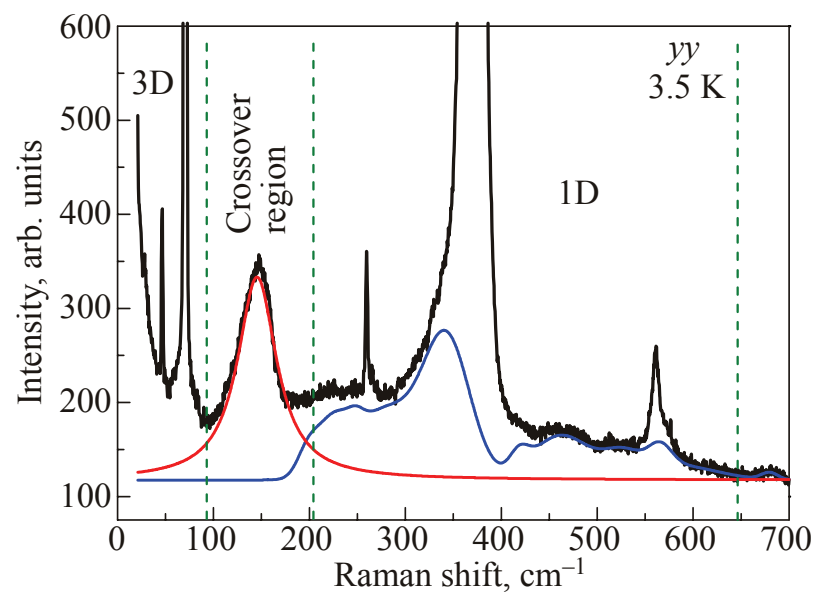

Fig. 7. Multi-spinon continuum at $T=3.5 \mathrm{~K}$ together with the longitudinal mode located at $145 \mathrm{~cm}^{-1}$ extracted from the $y y \mathrm{Ra}-$ man spectra of $\mathrm{KCuF}_{3}$. Dashed lines separate the different regimes of $\mathrm{KCuF}_{3}$ as a function of energy [9]. tigations [47-49], we assume magnetic excitations and the corresponding multi-spinon Raman-scattering processes as the origin of the observed background. The magnetic background scattering in RS is bounded by the upper and lower boundaries consistent with the boundaries of the multi-spinon continuum $(26 \mathrm{meV}<E<80 \mathrm{meV})$ determined in earlier investigations [9,47-49].

Below $T_{N}$ two new features emerge in different polarizations with different shapes and peak intensities, that can be attributed to magnetic excitations. Firstly, a broad band extending from about 100 to $180 \mathrm{~cm}^{-1}$, appears in the $x x$ and $y y$ polarization configurations with the incoming and outgoing light polarization perpendicular to the $c$ axis of the crystal (Fig. 8). Secondly, sharp and rather symmetric peak develops in crossed $y z$ polarization at around $113 \mathrm{~cm}^{-1}$ (Fig. 9). Both features appear below the magnetic ordering temperature, thus suggesting magnetic excitations as their origin. We attribute the broad band to a longitudinal mode, and the sharp peak with lower energy to a transverse mode. This interpretation is motivated by the energy scale [9] of the peak energy and the selection rules.

In the following, we will discuss the above-mentioned observations in more details:

(i) Many theoretical and experimental investigations have been directed towards the spectroscopic investigations of the spinon continuum in the $1 \mathrm{D}$ spin- $1 / 2$ systems [50-53]. The multi-spinon excitation spectrum is observed

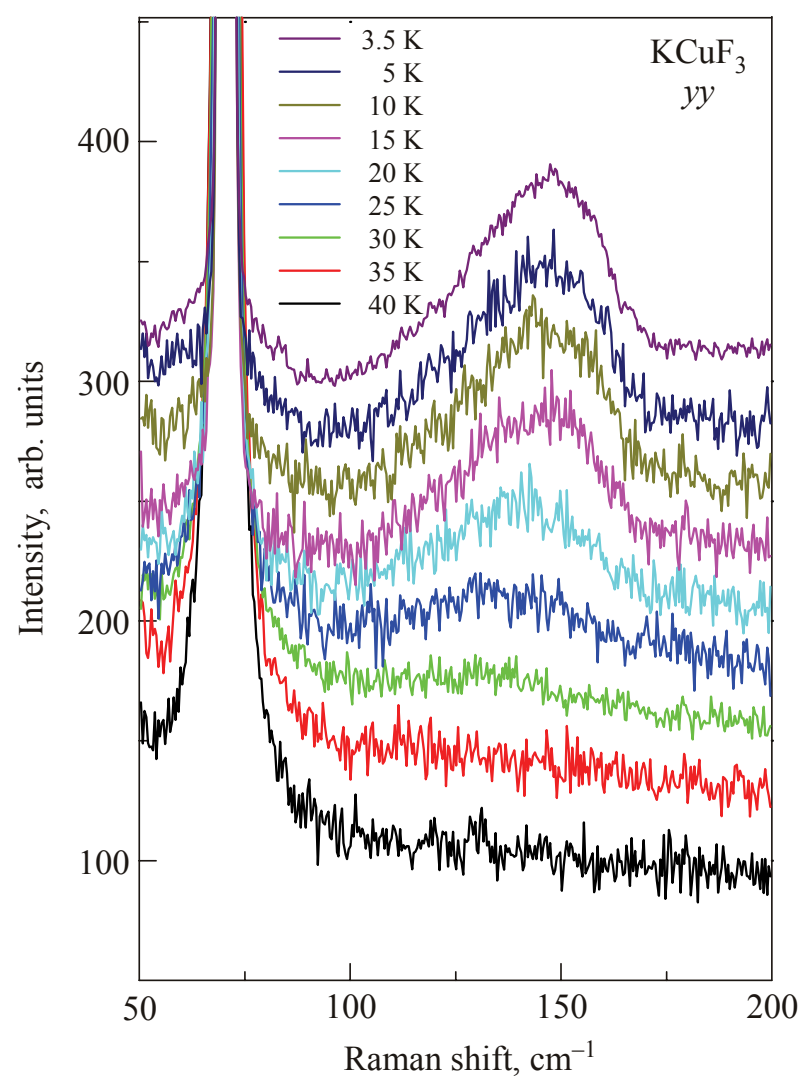

Fig. 8. Temperature dependence of the longitudinal mode in the Raman spectra of $\mathrm{KCuF}_{3}$. The spectra have been offset vertically for clarity. 


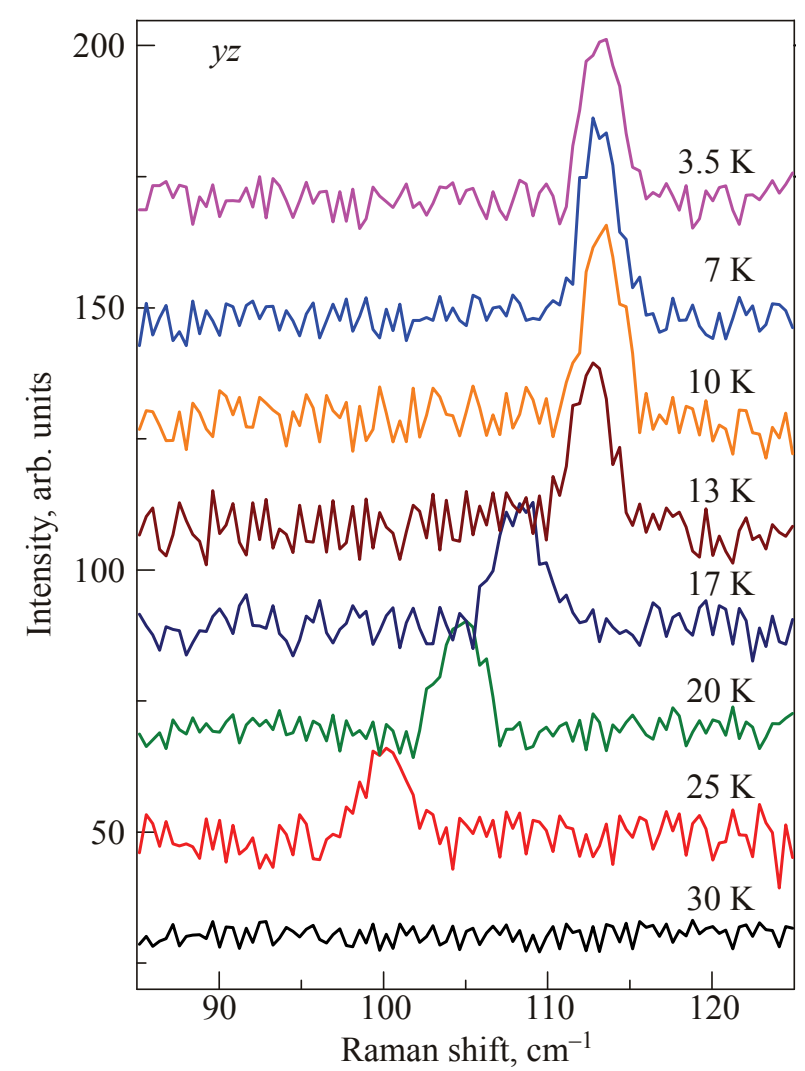

Fig. 9. Temperature dependence of the transverse mode in the Raman spectra of $\mathrm{KCuF}_{3}$. The spectra have been offset vertically for clarity.

in both low- and high-temperature phases of $\mathrm{KCuF}_{3}$ in neutron scattering experiments $[48,49,54]$ and was compared to the Müller ansatz [55], an analytic expressions for the two-spinon contribution of the Bethe ansatz solutions [56]. An excellent agreement was found for the temperatures above $T_{N}$. However, within the Fleury-Loudon scheme $[57,58]$ there would be no magnetic light scattering in the case of the strictly 1D linear chain with pure nearestneighbor interactions. The light scattering operator commutes with the original Heisenberg Hamiltonian. This is exactly the case for $\mathrm{KCuF}_{3}$, where only quasielastic scattering is observed in intrachain scattering configuration at temperatures above $T_{N}$. As it is clearly seen in Fig. 7, a wide scattering continuum presents in Raman spectra of $\mathrm{KCuF}_{3}$ at temperatures below $T_{N}$ where long-range $3 \mathrm{D}$ magnetic order develops.

The low-temperature spin-excitation continuum in $\mathrm{KCuF}_{3}$, observed in inelastic neutron scattering experiments, was compared to the theoretical predictions both for two-magnon excitations in a 3D antiferromagnet and for multi-spinon excitations in a 1D AFM spin-1/2 chain [54]. It was found that conventional 3D spin-wave theory with the inclusion of two-magnon terms could qualitatively explain the observations at low energies but not the continuum at higher energies.
It is known that the ordered moment per $\mathrm{Cu}$ site in $\mathrm{KCuF}_{3}$ is reduced by $50 \%$ at $T<<T_{N}$ compared to the saturation value in $3 \mathrm{D}$ due to quantum fluctuations [25]. Therefore, this system clearly has aspects of both 1D and $3 \mathrm{D}$ spin-1/2 magnetism at low temperatures. The question arises what happens to the excitation spectrum when weak interchain coupling is "switched on". A simple physical picture was provided by the chain-mean field theory [59], treating the interchain interactions as a staggered field and applying a random phase approximation [60,61]. A multispinon continuum starting at $\sim 22 \mathrm{meV}$ and extending upward in energy was predicted [61]. We want to highlight that the observations of a structured background in our RS experiments at low temperatures are consistent with an interpretation of the magnetic continuum in terms of multispinon excitations: 1) in energy position and 2), most surprisingly, the shape of the multi-spinon background is similar to the spin excitation continuum measured using neutron scattering [48]. As to the polarization properties, inclusion of the terms beyond the nearest neighbors in $c$ direction into the effective light coupling Hamiltonian, spin-orbit coupling, and "phonon assisted superexchange" [62] can lead to the appearance of multi-spinon scattering not only in a polarization parallel to the chain direction [57].

(ii) The existence of a novel longitudinal mode in coupled quantum chain compounds has been established theoretically and experimentally $[9,10,60,64,65]$. This mode appears in the AFM ordered phase of spin-1/2 quasi-onedimensional system where a long-range order coexists with strong quantum fluctuations. Furthermore it indicates a reduction in the ordered spin moment of the spin-1/2 antiferromagnet. In theory, the longitudinal mode in $\mathrm{KCuF}_{3}$ is predicted at $17.4 \mathrm{meV}$ [61] and observed in neutron scattering at $16 \mathrm{meV}$ [65] with a width of $5 \mathrm{meV}$. In our Raman scattering experiments the longitudinal mode (Fig. 8) is observed in $x x$ and $y y$ scattering geometry at $145 \mathrm{~cm}^{-1}(18 \mathrm{meV})$ at $T=3.5 \mathrm{~K}$ with a width of $\sim 5 \mathrm{meV}$. With increasing temperatures the peak position of this mode shifts to $128 \mathrm{~cm}^{-1}(15.8 \mathrm{meV})$ and it broadens up to $16.6 \mathrm{meV}$ in the vicinity of $T_{N}$. The intensity of the longitudinal mode is plotted as a function of temperature in Fig. 10. The energy of the longitudinal mode is to a good approximation proportional to the square of the ordered moment and this is in good agreement with neutron experiments [10].

(iii) Inelastic light scattering in a homogeneous spin chain based on the Heisenberg model does not allow the observation of transverse modes known for $T<T_{N}$ from spin-wave theory [66]. Therefore we have to assign the observation of a sharp mode in $y z$ crossed geometry at $113 \mathrm{~cm}^{-1}$ (see Fig. 9) to modifications of the scattering mechanism. As candidates there exist interchain interaction, spin-orbit interaction, or orbital fluctuations. While interchain interaction is certainly present and a key factor for the observation of long-range order, it would by itself 


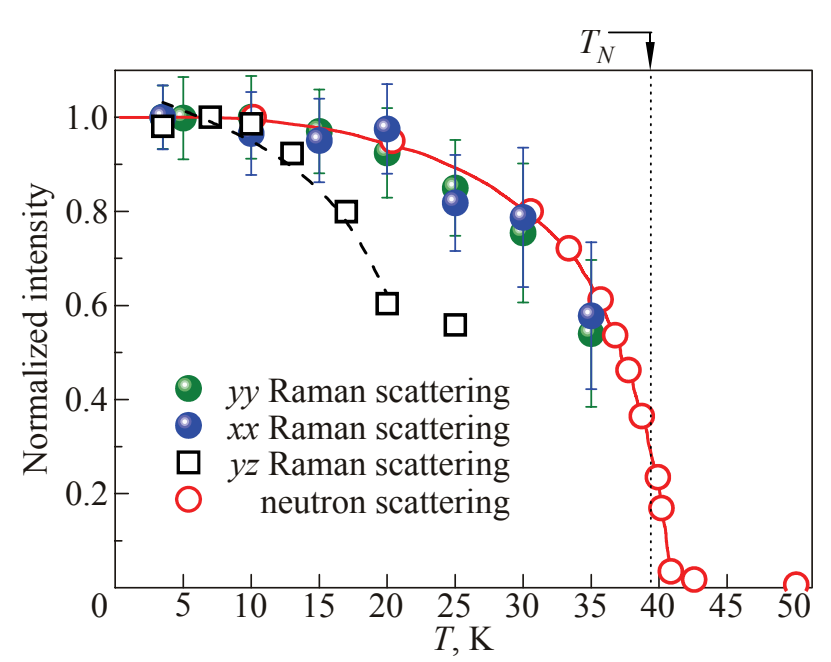

Fig. 10. Temperature dependence of the normalized integrated intensity of the longitudinal mode obtained from Raman (closed circles) and neutron scattering (open circles) [10] experiments on $\mathrm{KCuF}_{3}$. The open squares present the temperature dependence of the normalized intensity of the transverse mode and the black dashed line show the fit with a power law.

not lead to a large scattering intensity by spin flips. Therefore and in agreement with the scattering in crossed geometry, spin-orbit interaction may be the origin of this mode. Comparing the temperature variation of the intensity of the transverse and longitudinal modes (Fig. 10), it can be seen that the former does not trace the Néel temperature of $39 \mathrm{~K}$. It rather fits to a power law, $I=I_{0}\left(1-T / T_{N}\right)^{2 \beta}$, with $T_{N}=22 \mathrm{~K}$ and $\beta=0.217$. This variation points to another origin given in a small amount of the second structural polytype $\left(D_{4 h}^{5}\right)$ of $\mathrm{KCuF}_{3}$ phase presence in our sample.

Generally speaking, the magnetic excitation spectrum of $\mathrm{KCuF}_{3}$ collected in Raman scattering measurements shows the features characteristic for the $1 \mathrm{D}$ behavior over a large range of energies and temperatures. At low temperatures (below $T_{N}$ ) additional low-energy excitations are found. From these the longitudinal mode, a quantum feature, which arises in systems where a limited amount of long-range magnetic order co-exist with quantum fluctuations, is the most interesting as it separates the energy range of 1D- and 3D-like magnetic excitations at $T<T_{N}$. Our observations are in an excellent agreement with neutron scattering data $[9,10,48,54]$ and strongly support the concept that $\mathrm{KCuF}_{3}$ is a good realization of a material close to a quantum critical point.

\section{Summary}

In summary, the main outcomes of this work are the following:

(i) Temperature-dependent Raman spectra of single crystalline $\mathrm{KCuF}_{3}$ show a strong softening of the lowestlying $E_{g}(1)$ and the $B_{1 g}$ mode for $T>T_{S}$. Both modes, to- gether with the $E_{g}(2)$ mode (at about $260 \mathrm{~cm}^{-1}$ ) exhibit anomalies at the characteristic temperature $T_{S}=50 \mathrm{~K}$. In $x z$ scattering configuration the $E_{g}(2)$ doublet clearly splits by about $6 \mathrm{~cm}^{-1}$. The temperature dependence of the linewidth of these modes yields an activated behavior with an energy $U_{r} \approx 50 \mathrm{~K}$ corresponding to $T_{S}$. We ascribe this anomalous behavior to a simultaneous and coupled change in magnetic-structural-orbital sectors at cooling. An antiferrodistortive lattice instability is connected with strong dynamic displacements of the $\mathrm{F}^{-}$ions away from the $\mathrm{Cu}-\mathrm{F}-\mathrm{Cu}$ line along the $c$ axis. The displacements are strongly influencing the spin-spin relaxation by allowing for a Dzyaloshinsky-Moriya interaction. They become static for $T<T_{S}$.

(ii) The temperature-dependent magnetic excitation spectrum of $\mathrm{KCuF}_{3}$ was collected in our Raman scattering experiment for the first time. In the high-temperature phase it shows quasielastic scattering, characteristic for the 1D chain systems. In the low-temperature phase, below $T_{N}$, transverse and longitudinal magnetic modes as well as multi-spinon continuum emerges in different energy regions of the Raman spectra in different polarizations and with different shapes and intensities. The simultaneous observation of these magnetic excitations evidences the coexistence of a limited amount of long-range antiferromagnetic order with strong quantum fluctuations in $\mathrm{KCuF}_{3}$.

\section{Acknowledgments}

We like to thank M.V. Eremin and B. Lake for important discussions. D.W. and P.L. acknowledges support by B-IGSM and NTH "Contacs in Nanosystems". We also acknowledge support by the DFG via TRR80 and via LE 967/6-1 and the Swiss NSF through NCCR MaNEP.

1. S. Kadota, I. Yamada, S. Yoneyama, and K. Hirakawa, $J$. Phys. Soc. Jpn. 23, 751 (1967).

2. K.I. Kugel and D.I. Khomskii, Sov. Phys. Usp. 25(4), 231 (1982).

3. A.I. Liechtenstein, V.I. Anisimov, and J. Zaanen, Phys. Rev. B52, R5467 (1995).

4. J.E. Medvedeva, M.A. Korotin, V.I. Anisimov, and A.J. Freeman, Phys. Rev. B65, 172413 (2002).

5. I. Leonov, N. Binggeli, Dm. Korotin, V.I. Anisimov, N. Stojic, and D. Vollhardt, Phys. Rev. Lett. 101, 096405 (2008).

6. I. Leonov, Dm. Korotin, N. Binggeli, V.I. Anisimov, and D. Vollhardt, arXiv:0909.1283 (unpublished).

7. E. Pavarini, E. Koch, and A.I. Lichtenstein, Phys. Rev. Lett. 101, 266405 (2008)

8. A. Okazaki and Y. Suemune, J. Phys. Soc. Jpn. 16, 671 (1961).

9. B. Lake, D.A. Tennant, C.D. Frost, and S.E. Nagler, Nature Mat. 4, 329 (2005). 
10. B. Lake, D.A. Tennant, and S.E. Nagler, Phys. Rev. B71, 134412 (2005).

11. A. Okazaki and Y. Suemune, J. Phys. Soc. Jpn. 16, 176 (1961).

12. R.H. Buttner, E.N. Maslen, and N. Spadaccini, Acta Cryst. B46, 131 (1990).

13. M. Tsukuda and A. Okazaki, J. Phys. Soc. Jpn. 33, 1088 (1972).

14. M.T. Hutchings, E.J. Samuelson, G. Shirane, and K. Hirakawa, Phys. Rev. 188, 919 (1969).

15. M.T. Hutchings, H. Ikeda, and J.M. Milne, J. Phys. C: Solid State Phys. 12, L739 (1979).

16. S.K. Satija, J.D. Axe, G. Shirane, H. Yoshizawa, and K. Hirakawa, Phys. Rev. B21, 2001 (1980).

17. M. Hidaka, T. Eguchi, and I. Yamada, J. Phys. Soc. Jpn. 67, 2488 (1998).

18. T. Ueda, K. Sugawara, T. Kondo, and I. Yamada, Solid State Commun. 80, 801 (1991).

19. I. Yamada, H. Fujii, and M. Hidaka, J. Phys.: Condens. Matter 1, 3397 (1989).

20. C. Mazzoli, G. Allodi, R. De Renzi, G. Guidi, and P. Ghigna, J. Magn. Magn. Mater. 242, 935 (2002).

21. L. Li, Q. Shi, M. Mino, H. Yamazaki, and I. Yamada, J. Phys.: Condens. Matter 17, 2749 (2005).

22. I. Yamada and N. Kato, J. Phys. Soc. Jpn. 63, 289 (1994).

23. N. Binggeli and M. Altarelli, Phys. Rev. B70, 085117 (2004).

24. L. Paolasini, R. Caciuffo, A. Sollier, P. Ghigna, and M. Altarelli, Phys. Rev. Lett. 88, 106403 (2002).

25. R. Caciuffo, L. Paolasini, A. Sollier, P. Ghigna, E. Pavarini, J. van den Brink, and M. Altarelli, Phys. Rev. B65, 174425 (2002).

26. M.V. Eremin, D.V. Zakharov, H.-A. Krug von Nidda, R.M. Eremina, A. Shuvaev, A. Pimenov, P. Ghigna, J. Deisenhofer, and A. Loidl, Phys. Rev. Lett. 101, 147601 (2008).

27. J. Deisenhofer, I. Leonov, M.V. Eremin, Ch. Kant, P. Ghigna, F. Mayr, V.V. Iglamov, V.I. Anisimov, and D. van der Marel, Phys. Rev. Lett. 101, 157406 (2008).

28. A.E. Nikiforov and S.Yu. Shashkin, Phys. Solid State 38, 1880 (1996).

29. K. Wakamura and T. Arai, J. Appl. Phys. 63, 5824 (1988).

30. P.G. Klemens, Phys. Rev. 148, 845 (1966).

31. Balkanski, R.F. Wallis, and E. Haro, Phys. Rev. B28, 1928 (1983).

32. G.A. Samara and P.S. Peercy, Phys. Rev. B7, 1131 (1973).

33. D.J. Lockwood, Fiz. Nizk. Temp. 28, 709 (2002) [Low Temp. Phys. 28, 505 (2002)].

34. D.J. Lockwood, R.S. Katiyar, and V.C.Y. So, Phys. Rev. B28, 1983 (1983).

35. D.J. Lockwood, Proc. IX Intern. Conference on Raman Spectroscopy, M. Tsuboi (ed.), Chem. Soc. Jpn., Tokyo (1984), p. 810.

36. W. Baltensperger and J.S. Helman, Helv. Phys. Acta 41, 668 (1968).

37. W. Baltensperger, J. Appl. Phys. 41, 1052 (1970).
38. P. Brüesch and F.D. Ambrogio, Phys. Status Solidi B50, 513 (1972).

39. D.J. Lockwood and M.G. Cottam, J. Appl. Phys. 64, 5876 (1988).

40. J.M. Wesselinowa and A.T. Apostolov, J. Phys.: Condens. Matter 8, 473 (1996).

41. J.C.T. Lee, S. Yuan, S. Lal, Y.I. Joe, Yu Gan, S. Smadici, K. Finkelstein, Y. Feng, A. Rusydi, P.M. Goldbart, S.L. Cooper, and P. Abbamonte, arXiv:0911.0619; Nature Physics doi:10.1038/nphys2117 (2011).

42. K.-Y. Choi, Yu.G. Pashkevich, V.P. Gnezdilov, G. Güntherodt, A.V. Yeremenko, D.A. Nabok, V.I. Kamenev, S.N. Barilo, S.V. Shiryaev, A.G. Soldatov, and P. Lemmens, Phys. Rev. B74, 064406 ( 2006).

43. J. Deisenhofer, M.V. Eremin, D.V. Zakharov, V.A. Ivanshin, R.M. Eremina, H.-A. Krug von Nidda, A.A. Mukhin, A. Balbashov, and A. Loidl, Phys. Rev. B65, 104440 (2002).

44. T. Rudolf, Ch. Kant, F. Mayr, J. Hemberger, V. Tsurkan, and A. Loidl, Phys. Rev. B76, 174307 (2007).

45. P. Lemmens, G. Güntherodt, and C. Cros, Phys. Rep. 375, 1 (2003) and references therein.

46. I. Yamada and H. Onda, Phys. Rev. B49, 1048 (1994).

47. S.E. Nagler, D.A. Tennant, R.A. Cowley, T.G. Perring, and S.K. Satija, Phys. Rev. B44, 12361 (1991).

48. D.A. Tennant, R.A. Cowley, S.E. Nagler, and A.M. Tsvelik, Phys. Rev. B52, 13368 (1995).

49. D.A. Tennant, T.G. Perring, R.A. Cowley, and S.E. Nagler, Phys. Rev. Lett. 70, 4003 (1993).

50. H.-J. Mikeska and A.K. Kolezhuk, in Quantum Magnetism, Lecture Notes in Physics, vol. 645, U. Schollwock, J. Richter, B.J.J. Farell, and R.F. Bishop (eds.), Springer-Verlag, Berlin (2004), p. 1, and references therein.

51. P. Lemmens, G. Guntherodt, and C. Gross, Phys. Rep. 375, 1 (2003); P. Lemmens and P. Millet, in: Quantum Magnetism, Lecture Notes in Physics, vol. 645, U. Schollwock, J. Richter, B.J.J. Farell, and R.F. Bishop (eds.), Springer-Verlag, Berlin (2004), and references therein.

52. A. Gozar and G. Blumberg, in: Frontiers in Magnetic Materials, A.V. Narlikar (ed.), Springer-Verlag, Berlin (2005), p. 697 , and references therein.

53. A.A. Zvyagin and V.O. Cheranovskii, Fiz. Nizk. Temp. 35, 578 (2009) [Low Temp. Phys. 35, 455 (2009)].

54. D.A. Tennant, S.E. Nagler, D. Welz, G. Shirane, and K. Yamada, Phys. Rev. B52, 13381 (1995).

55. G. Muller, H. Thomas, H. Beck, and J.C. Bonner, Phys. Rev. B24, 1429 (1981).

56. H. Bethe, Z. Phys. 71, 205 (1931).

57. P.A. Fleury and R. Loudon, Phys. Rev. 166, 514 (1968).

58. B.S. Shastry and B.I. Shraiman, Phys. Rev. Lett. 65, 1068 (1990); B.S. Shastry and B.I. Shraiman, Int. J. Mod. Phys. B5, 365 (1991).

59. D.J. Scalapino, Y. Imry, and P. Pincus, Phys. Rev. B11, 2042 (1975).

60. H.J. Schulz, Phys. Rev. Lett. 77, 2790 (1996).

61. F.H.L. Essler, A.M. Tsvelik, and G. Delfino, Phys. Rev. B56, 11001 (1997). 
62. B.S. Shastry and B.I. Shraiman, Phys. Rev. Lett. 65, 1068 (1990).

63. R.R.P. Singh, P. Prelovšek, and B.S. Shastry, Phys. Rev. Lett. 77, 4086 (1996).

64. B. Lake, R.A. Cowley, and D.A. Tennant, J. Phys.: Condens. Matter 9, 10951 (1997).
65. B. Lake, D.A. Tennant, and S.E. Nagler, Phys. Rev. Lett. 85, 832 (2000).

66. For a review see: M.G. Cottam and D.J. Lockwood, Light Scattering in Magnetic Solids, Wiley, New York (1986). 